\title{
Utilizing Correlative, Multi-Technique Surface Analysis to Complement Microscopy Studies
}

\author{
Tim Nunney ${ }^{1}$, Christopher Deeks ${ }^{1}$, Paul Mack ${ }^{1}$, Robin Simpson ${ }^{1}$ and Chris Stephens ${ }^{1}$ \\ 1. Thermo Fisher Scientific, East Grinstead, West Sussex, UK.
}

Full chemical characterization of a sample necessitates measurement of both bulk and surface properties. To achieve this, an array of experimental techniques may be employed; allowing the field of view to be narrowed both in the $\mathrm{X}-\mathrm{Y}$ plane, to investigate changes in lateral surface morphology, and in $\mathrm{Z}$ to enable the understanding of varying surface and interfacial chemistry with depth. If it is easy to correlate the data, then building a comprehensive picture of the nature of the sample is possible.

To this end we have used a new, multi-technique surface analysis platform that incorporates microfocusing X-ray photoelectron spectroscopy (XPS), ion scattering spectroscopy (ISS), reflected electron energy loss spectroscopy (REELS), UV photoelectron spectroscopy (UPS) and Raman spectroscopy to complement imaging analysis completed using EDS and EBSD. The ability to combine high spatial resolution imaging in the $\mathrm{X}-\mathrm{Y}$ plane, with highly surface sensitive analysis on the new platform.

An example of this approach is shown in figure 1, where a CIGS solar cell device is analyzed using EDS and XPS. The same areas on the surface were analyzed. Areas 1 and 2 are intact areas of the active material, area 3 is an area that has the electrical contact, and area 4 is an area that has delaminated. Compositional analysis shows that the surface measured by XPS, and comprising the outer $10 \mathrm{~nm}$ of the sample, is markedly different from the composition determined using EDS. The presence of an indium tin oxide overcoat is shown by XPS, which is too thick to be easily detected via EDS. Comparison of cross-sectional images from the SEM with depth profile analysis from the same device allows accurate determination of the thickness of layers, the compositional variation with the layers (particularly important for the CIGS layer), and establishing exactly where the delamination occurs.

In this presentation it will be shown how this approach is beneficial using additional examples from a range of sample types, including carbon nanomaterials, microelectronics samples, and the analysis of artworks. 


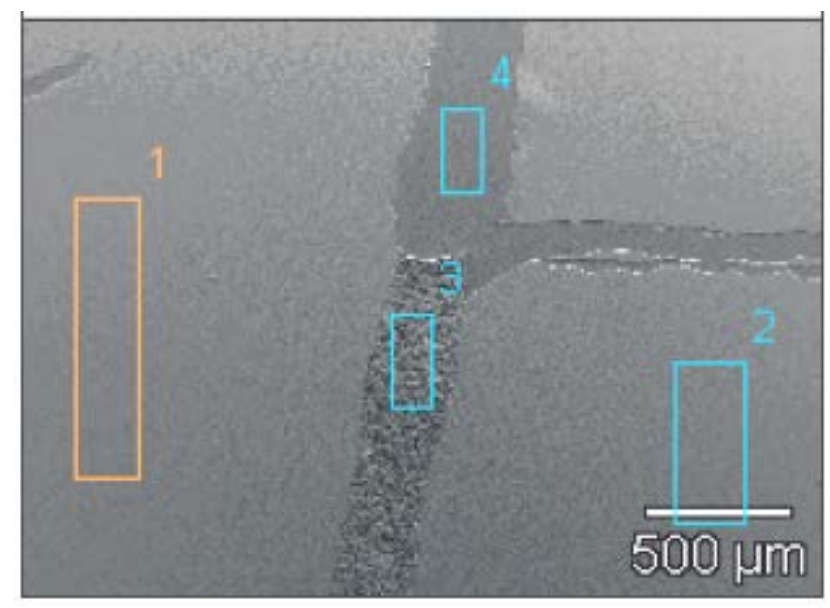

Figure 1. SEM image of the CIGS solar cell sample showing the 4 areas analyzed.

\begin{tabular}{|c|c|c|c|c|c|c|c|c|c|c|c|}
\hline EDS & Ga-K & Zn-K & O-K & Cr-K & In-L & Cd-L & Ag-L & Mo-L & Se-K & Fe-K & Cu-K \\
\hline Area 1 & 2.9 & 2.5 & 17.4 & 0.8 & 24.3 & 7.4 & & 2.3 & 22.9 & 2.4 & 12.4 \\
\hline Area 2 & 3.0 & 2.9 & 17.6 & 0.7 & 25.0 & 7.8 & & 2.1 & 21.6 & 2.5 & 12.3 \\
\hline Area 3 & & & & & & & 100.0 & & & & \\
\hline Area 4 & & & & 17.7 & & & & 61.0 & 2.9 & 18.4 & \\
\hline
\end{tabular}

Table 1. Measured composition by EDS for the 4 indicated areas.

\begin{tabular}{|c|c|c|c|c|c|c|c|c|c|}
\hline XPS & Ga2p3 & Zn2p3 & O1s & Sn3d5 & In3d & Cd3d5 & Ag3d & Mo3d & Se3d \\
\hline Area 1 & 0.3 & 2.5 & 68.2 & 2.7 & 26.0 & 0.2 & 0.00 & 0.2 & 0.0 \\
\hline Area 2 & 0.0 & 2.5 & 66.6 & 2.9 & 27.4 & 0.2 & 0.1 & 0.1 & 0.2 \\
\hline Area 3 & 0.0 & 1.8 & 65.2 & 2.6 & 24.3 & 0.1 & 5.5 & 0.5 & 0.1 \\
\hline Area 4 & 0.1 & 1.4 & 41.1 & 1.2 & 11.6 & 0.0 & 0.0 & 17.1 & 26.7 \\
\hline
\end{tabular}

Table 2. Measured composition by XPS for the 4 indicated areas. 